\title{
Googling Alzheimer Disease: An Infodemiological and Ecological Study
}

\author{
Bernadeth Lyn C. Piamonte ${ }^{a}$ Veeda Michelle M. Anlacan ${ }^{a, b}$ \\ Roland Dominic G. Jamora ${ }^{a}$ Adrian I. Espiritu ${ }^{a, c, d}$
}

aDepartment of Neurosciences, College of Medicine and Philippine General Hospital, University of the Philippines Manila, Manila, Philippines; ${ }^{b}$ Center for Memory and Cognition, Department of Neurosciences, Philippine General Hospital, University of the Philippines Manila, Manila, Philippines; ' ${ }^{C}$ epartment of Clinical Epidemiology, College of Medicine, University of the Philippines Manila, Manila, Philippines; ${ }^{\mathrm{d} D i v i s i o n}$ of Neurology, Department of Medicine, St. Michael's Hospital, University of Toronto, Toronto, ON, Canada

\section{Keywords}

Alzheimer disease - Search volume index - Google Trends . Infodemiology

\begin{abstract}
Introduction: Understanding the emergent role of the internet on the health-seeking behavior of people is critical not only in the areas of medicine and public health but also in the field of infodemiology. Methods: Using Google Trends, data on global search queries for Alzheimer disease (AD) between January 2004 and April 2021 were analyzed. The relationship between online interest, as reflected by search volume index (SVI), and measures of disease burden, namely prevalence, deaths, and disability-adjusted life years, was evaluated. Results: There was a reduction in the tendency to search for AD during the past two decades. SVI peaks corresponded to news of famous people with AD and awareness months. Symptoms, causes, and differences with the term dementia were central queries for persons interested in AD. No notable overall correlation between SVI and measures of disease burden was found due to competing results. Subgroup analyses, however, showed that these correlations may be influenced by socioeconomic development, with strong negative significant associations observed in lower
\end{abstract}

middle-income countries. Conclusion: Online interest in AD may represent a more complex metric influenced by socioeconomic factors. Awareness of the impact of celebrity diagnosis and awareness months on online search behavior may prove useful in the planning of public health campaigns for AD.

(c) 2021 The Author(s). Published by S. Karger AG, Basel

\section{Introduction}

The internet has been an increasingly common source of online health-related information for millions of people. Public online interest in health and diseases can be reflected by trends in popular web search engines, such as Google [1]. Analysis of these trend data allows for realtime surveillance and ultimately serves to inform public health and policy [2]. This type of research constitutes the discipline of infodemiology: the science of determinants and distribution of information, especially on the internet [3].

Bernadeth Lyn C. Piamonte and Veeda Michelle M. Anlacan are joint first authors.
C) 2021 The Author(s).

Published by S. Karger AG, Basel

This is an Open Access article licensed under the Creative Commons Attribution-NonCommercial-4.0 International License (CC BY-NC) (http://www.karger.com/Services/OpenAccessLicense), applicable to the online version of the article only. Usage and distribution for commercial purposes requires written permission.
Correspondence to:

Bernadeth Lyn C. Piamonte, bcpiamonte@up.edu.ph

Adrian I. Espiritu, espirituadrian@gmail.com 
Dementia poses significant challenges to patients, caregivers, and healthcare systems due to its rising prevalence and socioeconomic burden [4]. Alzheimer disease (AD) is the prototype and accounts for approximately $50-60 \%$ of all dementias [5]. In helping meet these significant challenges, current research into better diagnostics and treatments continues to advance and has been very promising. As more and more research and newer findings about $\mathrm{AD}$ emerge, communication in the new age of information technology is vital and imperative in decision-making. Older adults are increasingly online, with $73 \%$ of Americans aged 65 years or older say they use the internet as of 2019 [6]. Analysis of Google searches about dementia has shown an increase over time in some countries and others have suggested that search volume data temporally coincided with the number of local new dementia cases $[7,8]$. The aim of this study was to evaluate Google search trends worldwide over time for $\mathrm{AD}$ and assess the correlation between public online interest in $\mathrm{AD}$ and measures of the disease burden associated with $\mathrm{AD}$ and other dementias.

\section{Methodology}

\section{Google Trends Data Availability}

Google Trends is a popular open tool that provides online traffic data on selected keywords and topics over time. Data analyses generated from Google Trends are being increasingly utilized in healthcare research [9]. Search activity is reported as search volume index (SVI), which is reflective of data that are normalized over a specific time period and geographic location to improve comparability between search terms. The numbers on the graphs generated reflect the number of searches completed using Google for a specific term relative to the total number of searches completed over time. They are scaled on a range of $0-100$, where 100 is the maximum search interest for the time and location specified. A score of 0 means there were not enough data for this term.

\section{Search Process and Data Retrieval}

Online interest in AD was analyzed using Google Trends. The keyword "Alzheimer disease" or "Alzheimer's disease" was entered in the Google Trends main page (available at: http://www.google. com/trends, accessed 13 May 2021) [10]. The search phrase "Alzheimer's disease" yielded higher SVIs compared to "Alzheimer disease" and was hence the more commonly searched phrase. We opted to use "Alzheimer's disease (Disease)" than "Alzheimer's disease (search term)" as the former is inclusive of all search topics related to $\mathrm{AD}$ and not just search queries that include the selected text [1]. We set the following filters for the analyzed queries: "Worldwide," in "All categories" and for "Web searches." To reduce the noise in the data, we kept the default Google Trends option to exclude low search volume regions. We collected SVIs on a month-by-month basis dating from January 1, 2004, the earliest time with available data from Google Trends, to April 30, 2021. Data retrieval complied with a previously published Checklist for Documentation of Google Trends [9].

Data Analysis

We descriptively analyzed the worldwide changes over time of search queries. We checked for seasonality in the time trend and possible correlations with major world events reported in Google Trends. We also reviewed top and rising queries related to AD. Information on the prevalence, deaths, and disability-adjusted life years (DALYs) associated with $\mathrm{AD}$ and other dementias reported per region were collected from the published article by the Global Burden of Disease 2016 Dementia Collaborators [11]. To evaluate the relationship between online interest in $\mathrm{AD}$, as reflected by SVI, and these measures of disease burden, we performed a series of Spearman's rho correlation $(\rho)$. Sub-group analyses were also performed to accommodate the different regions and economic profiles involved, as classified by the World Bank [12]. A value of $p<$ 0.05 was considered statistically significant. Linear regression models and the creation of scattergram plots were also performed to determine the degree of variability contributed by the SVI in estimating disease burden. A trend line graph was created to determine the utility of SVI across the years. All statistical analyses were performed using the Stata 13 software.

\section{Ethical Considerations}

The infodemiology study does not include human subjects. Hence, the design of the study did not require Research Ethics Board approval. The study does not violate the terms of use of Google Trends.

\section{Results}

\section{Search Trends for $A D$}

Overall, worldwide online interest in $\mathrm{AD}$ decreased by $29 \%$ over the entire period between January 2004 and April 2021 as shown in Figure 1. A progressive decline in SVI over time was observed from January 2004 up until July 2013 , with a reduction by $63 \%$ compared to its peak $(S V I=100)$ at June 2004. By April 2021, online interest has picked up by $62 \%$ compared to its nadir (SVI $=37$ ) in July 2013. The SVI has remained to be within 49 and 69 worldwide and with an average of 59.57 (95\% CI: 57.8761.26).

The time trend revealed a general seasonal variation: the interest was higher during September and lower during July and December. Main SVI peaks for AD corresponded with major news and current events as follows: "President Ronald Reagan (40th president of the USA) loses fierce battle with Alzheimer's ..." (June 2004: SVI = 100) [13]; "Robertson (religious broadcaster of The $700 \mathrm{Club}$ ) stirs passions with suggestion to divorce an Alzheimer's patient" (September 2011: SVI = 60) [14]; “Biogen's Alzheimer's therapy (aducanumab) granted Food and Drug Administration 


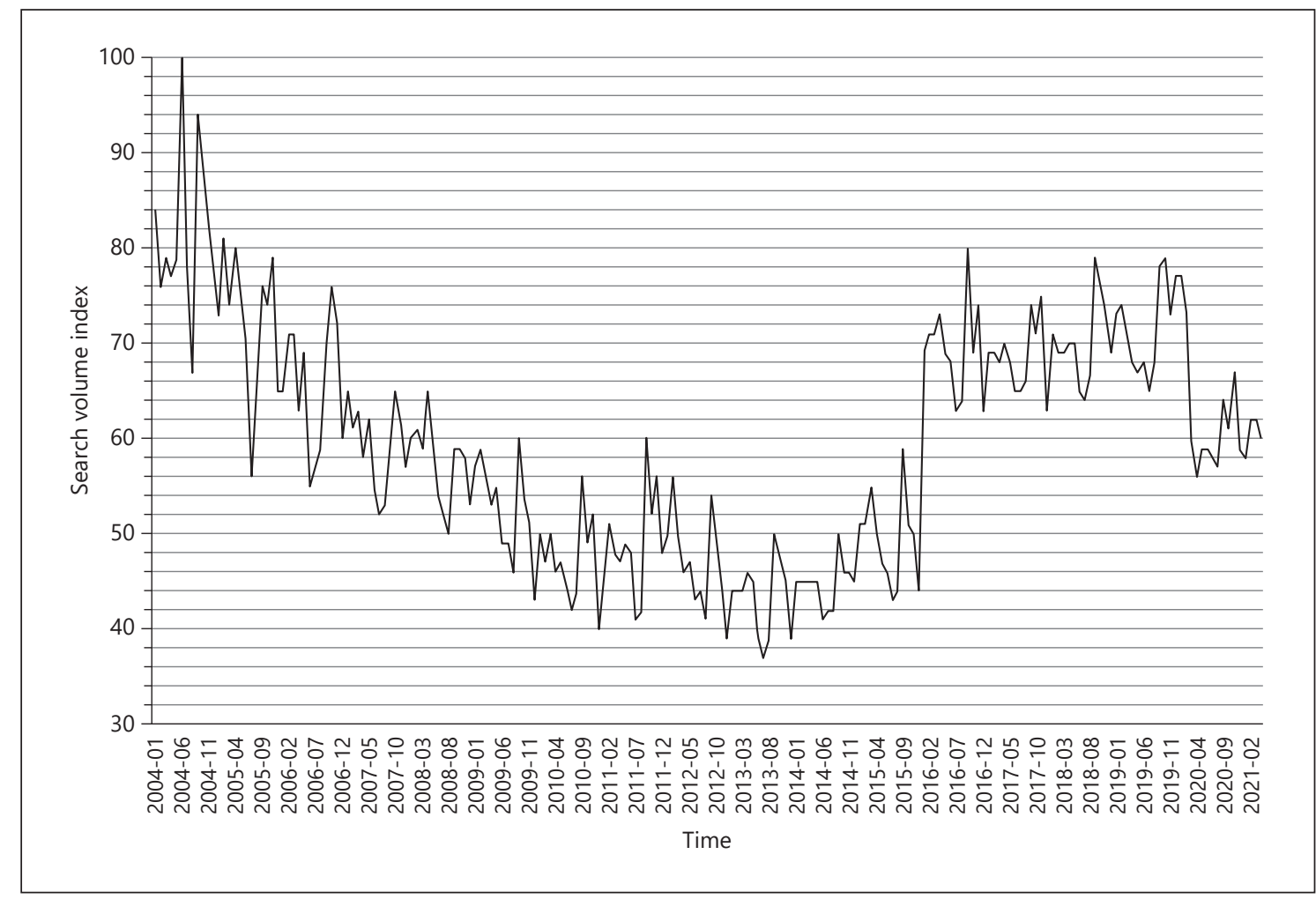

Fig. 1. SVIs for AD worldwide from January 2004 through April 2021. Data from Google Trends: interest over time.

fast-track status" (September 2016: SVI = 80) [15]; “How Biogen's discontinued Alzheimer's drug (aducanumab) got a second life" (October 2019: SVI = 79) [16]; and "B. Smith (American lifestyle guru) dies at 70 of early onset Alzheimer's" (February 2020: SVI = 73) [17]. On the other hand, a stark 23\% drop was observed after the World Health Organization formally declared COVID-19 a global pandemic in March 2020.

Top queries related to the Google search for AD included symptoms, causes, and differences with the term dementia. Search queries with the biggest increase over time included Russian, Chinese, Japanese, and Persian translations of terms related to dementia and $\mathrm{AD}$ [18]. As outlined in Table 1, the greatest search volume for AD was in Puerto Rico (SVI = 100), followed by Spain (SVI = $60)$, Chile (SVI $=53)$, Portugal (SVI $=53)$, and the Netherlands $(\mathrm{SVI}=51)$.

\section{Correlation between Online Interest and Measures of}

Disease Burden

The correlation between online interest in $\mathrm{AD}$ and the measures of disease burden associated with $\mathrm{AD}$, and oth- er dementias are presented in Table 2. There was no notable association as suggested by the correlation coefficients for the prevalence, deaths, and DALYs from AD. These correlation coefficients were also computed for the different regions and economic profiles included in the study - which showed variability across these locations and incomes. In the case of Europe and Central Asia - and to a certain extent Middle East and North Africa - the SVI increases along with the burden of disease. On the other hand, inverse correlation was notable for Latin America and the Caribbean and East Asia and Pacific - where the burden of disease was higher when the SVI was low. The correlation coefficients for the sub-Saharan African, South Asian, and North American regions could not be calculated because there were only one to two members in the regions listed. When the countries were classified based on their income, it was noted that only those with high incomes have a positive (nonsignificant) correlation between SVI and the burden of disease and the opposite for the lower and upper middle-income countries. There was a strong negative significant correlation between the SVI for the lower middle-income countries and the prev- 
Table 1. Ranked results of search volume analysis for $A D$ by region and the corresponding measures of disease burden associated with $\mathrm{AD}$ and other dementias

\begin{tabular}{|c|c|c|c|c|}
\hline \multirow[t]{2}{*}{ Region } & \multirow[t]{2}{*}{$\mathrm{SVI} \mathbf{I}^{*}$} & \multicolumn{3}{|c|}{ Measures of disease burden ${ }^{\dagger}$} \\
\hline & & prevalence & deaths & DALYs \\
\hline \multicolumn{5}{|c|}{ Highest online interest } \\
\hline Puerto Rico & 100 & 31,203 & 2,081 & 22,554 \\
\hline Spain & 60 & 830,915 & 57,098 & 561,948 \\
\hline Chile & 53 & 104,523 & 6,508 & 70,944 \\
\hline Portugal & 53 & 166,660 & 10,824 & 113,147 \\
\hline The Netherlands & 51 & 192,425 & 13,713 & 136,421 \\
\hline France & 51 & 877,760 & 65,775 & 611,185 \\
\hline Canada & 51 & 317,027 & 21,180 & 214,389 \\
\hline Italy & 51 & $1,370,308$ & 90,981 & 898,329 \\
\hline Finland & 49 & 83,950 & 6,686 & 66,130 \\
\hline USA & 46 & $4,029,450$ & 238,895 & $2,473,390$ \\
\hline \multicolumn{5}{|c|}{ Lowest online interest } \\
\hline Serbia & 17 & 76,985 & 4,305 & 52,068 \\
\hline Bulgaria & 16 & 76,346 & 4,140 & 51,189 \\
\hline Ukraine & 16 & 370,615 & 19,870 & 246,622 \\
\hline Egypt & 16 & 355,367 & 14,929 & 218,670 \\
\hline Thailand & 16 & 597,698 & 30,293 & 395,408 \\
\hline China & 13 & $10,427,487$ & 476,898 & $6,637,268$ \\
\hline Pakistan & 12 & 346,449 & 15,880 & 222,007 \\
\hline Indonesia & 12 & $1,111,081$ & 45,591 & 703,600 \\
\hline India & 11 & $2,933,814$ & 142,927 & $1,996,152$ \\
\hline Vietnam & 8 & 655,099 & 42,459 & 475,067 \\
\hline
\end{tabular}

DALYs, disability-adjusted life years. * Data from Google Trends: interest by region. ' Information on the "prevalence," "deaths," and "DALYs" for "Alzheimer disease and other dementias" was obtained from the published article by the Global Burden of Disease 2016 Dementia Collaborators and was reported as counts.

Table 2. Spearman correlational analysis between online interest and measures of disease burden across region and income

\begin{tabular}{|c|c|c|c|c|c|c|}
\hline Worldwide & -0.1414 & 0.25 & -0.0661 & 0.60 & -0.1297 & 0.60 \\
\hline North America $(n=2)$ & - & - & - & - & - & - \\
\hline Latin America and the Caribbean $(n=13)$ & -0.3007 & 0.32 & -0.3062 & 0.31 & -0.3200 & 0.29 \\
\hline Europe and Central Asia $(n=29)$ & 0.2422 & 0.21 & 0.3408 & 0.07 & 0.2688 & 0.16 \\
\hline Middle East and North Africa $(n=8)$ & 0.0952 & 0.82 & 0.2381 & 0.57 & 0.0952 & 0.82 \\
\hline East Asia and Pacific $(n=12)$ & -0.5594 & 0.06 & -0.5245 & 0.08 & -0.5594 & 0.06 \\
\hline South Asia $(n=2)$ & - & - & - & - & - & - \\
\hline \multicolumn{7}{|l|}{ Across income* } \\
\hline High income $(n=36)$ & 0.1915 & 0.26 & 0.2488 & 0.14 & 0.2138 & 0.21 \\
\hline Upper middle income $(n=21)$ & -0.2892 & 0.20 & -0.2566 & 0.26 & -0.2787 & 0.22 \\
\hline Lower middle income $(n=10)$ & -0.8293 & 0.01 & -0.8964 & 0.01 & -0.8476 & 0.01 \\
\hline
\end{tabular}

$p$ value is set at 0.05 . DALYs, disability-adjusted life years; GNI, gross national income. * Classification by the World Bank; economies are divided among income groups according to $2019 \mathrm{GNI}$ per capita, calculated using the World Bank Atlas method. The groups are low income, USD 1,035 or less; lower middle income, USD 1,036-4,045; upper middle income, USD 4,046-12,535; and high income, USD 12,536 or more. 
alence ( $\rho:-0.83,95 \%$ CI: -0.42 to -0.96$)$, deaths ( $\rho:-0.90$, 95\% CI: -0.61 to -0.98 ), and DALYs ( $\rho:-0.85,95 \%$ CI: -0.47 to -0.96$)$ attributed to AD.

The data gathered from Google Trends were noted to account for a small proportion of variability in the study data. The computed R-squared values (i.e., the coefficients of variation) indicated that the SVI only accounted for $2.15 \%, 1.13 \%$, and $2.22 \%$ of the variability in prevalence, deaths, and DALYs, respectively. There was also no strong and clear trend between SVI and these different measures of disease burden in any of the scattergram plots.

\section{Discussion}

In analyzing Google Trends data on $\mathrm{AD}$ from the period of January 2004 to April 2021, we found that worldwide online interest in $\mathrm{AD}$ has decreased during the past two decades. This may correspond to the decreasing temporal trends of dementia incidence - a 13\% drop per decade - in Europe and North America, which encompasses most of the highest-ranked countries in terms of SVI for $\mathrm{AD}$ [19]. The rising queries related to the Google search for $\mathrm{AD}$, in comparison, may reflect countries that have seen a recent increase in burden of disease, such as Japan and China [20,21]. While this study failed to find a notable overall correlation between SVI and measures of disease burden likely due to the competing results across regions and incomes, sub-group analyses suggest that such correlations may be influenced partly by socioeconomics. We observe that the higher the income of a country, the more that public online interest aligns with and may be indicative of the disease burden. This could perhaps be attributed to more resources available to the region to invest in education, greater access to technology, and effective public awareness campaigns. It is noteworthy that the top 10 regions with the highest SVIs for $\mathrm{AD}$ in this study were all high-income economies and 7 out of these 10 had a National Dementia Plan [22]. The opposite trend, wherein a higher burden of disease is associated with lower online interest, is observed in lower income economies, significantly and strongly in lower middle-income economies and nonsignificantly in upper middle-income economies. These findings perhaps serve as a reflection of the challenge in educating and raising awareness for $\mathrm{AD}$ as well as providing greater online access to health-related information where resources are limited and priorities are competing to begin with. This puts lower income economies at an even more vulnerable position as they are projected to bear most of the brunt of the rise in dementia cases in future years [23]. Nine of the lowest 10 regions are also bereft of a National Dementia Plan [22]. There are sparse data existing about online information-seeking behaviors specific to $A D$, and few studies have documented variable observations of the impact of socioeconomic development on online search volume [24-26]. To our knowledge, this is the first study analyzing Google search trends worldwide over time for $\mathrm{AD}$ and its correlation with measures of disease burden.

We also observed a relation between SVI peaks and news headlines, particularly about famous people with $\mathrm{AD}$. This finding is in line with previous studies that have seen similar links between celebrity diagnosis and increased public interest in neurological disorders [24,27]. Making use of this association in public health campaigns, either through photographs or other promotional multimedia, holds promise for more effective awareness programs on and stigma reduction related to $\mathrm{AD}$. The seasonal pattern of SVI peaks in September also matches the celebration of World Alzheimer's Month in September [28]. This finding is consistent with past documentation of awareness months generating interest of Google users [29]. We surmise that World Alzheimer's Month further motivates healthcare professionals and institutions to initiate public health campaigns on $\mathrm{AD}$. A slump in online search volume on $\mathrm{AD}$ during the month of December is compatible with previous reports citing that most people will prefer to spend this time celebrating the holidays with friends and family [29]. Likewise, the drop in interest observed after March 2020 can be attributed to hyperawareness and desire for more online information on COVID-19 and related novel concepts, such as social distancing and mask wearing, combined with a decline in health-seeking behavior by patients with underlying health risks made more vulnerable by a pandemic [30].

Commonly searched queries related to $\mathrm{AD}$ include information on its symptoms, causes, and difference with the term dementia, highlighting the level of basic knowledge of the general public and their priorities of concern. Roughly one in every three internet users has said that online health-related information has influenced their decision to visit a doctor [31]. In this context, necessary precautions must be taken to ensure that accurate, reliable, and updated information on $\mathrm{AD}$ is available online for both patients and their caregivers.

The study has several limitations. While arguably the most popular, Google is not the only web search engine and its share of search traffic varies across regions [32]. The study data may be affected by the nonrepresentative sampling bias inherent in the Google Trends service. Us- 
ers were anonymous in keeping with data confidentiality, but this makes it impossible to subject the sample to statistical analyses by demographic characteristics. Information was collected from people with internet access, therefore possibly omitting lower income groups, novice computer users, or those in areas with lower scorings in freedom of speech [1]. Total search volume per region for $\mathrm{AD}$ was unavailable through Google Trends and would have added supplementary comparison metrics aside from SVI. Moreover, the study data gathered from Google Trends only accounted for a small proportion of variability in the estimates of disease burden likely because of outliers in the dataset and significant confounding brought about by the competing results in the correlation coefficients. This study did not include the incidence of $\mathrm{AD}$ in its correlational analyses. However, given that numerous studies have reported the growing use of Google Trends data in outbreak surveillance, a future study opportunity would be to investigate possible relationships between the incidence of $\mathrm{AD}$ and Google search trends for $\mathrm{AD}$ [9]. Lastly, this study excluded low search volume regions that may be susceptible to irregular variation. However, by doing so, low-income countries were not captured in the dataset. Studies including low search volume regions in similar correlational analyses may add further insight into the impact of socioeconomics on online search behavior. Given these limitations, caution must be given in processing the data and interpreting these results. a reduction in the tendency to search for $\mathrm{AD}$ during the past two decades. While no notable overall correlation between SVI and measures of disease burden was found, sub-group analyses revealed that SVIs for AD may instead represent a more complex metric influenced by socioeconomic factors. News of celebrity diagnosis and line search behavior for $\mathrm{AD}$, and this knowledge may prove useful in planning public health campaigns for $\mathrm{AD}$. ment of Epidemiology and Biostatistics, College of Public Health, University of the Philippines Manila, Manila, Philippines, for sta-

\section{Statement of Ethics}

This study does not include human subjects. Hence, the design of the study did not require Research Ethics Board approval.

\section{Conflict of Interest Statement}

The authors have no conflicts of interest to declare.

\section{Funding Sources}

This research did not receive any specific grant from funding agencies in the public, commercial, or not-for-profit sectors.

\section{Author Contributions}

B.L.C.P., V.M.M.A., R.D.G.J., and A.I.E. conceived the idea. B.L.C.P. wrote the initial drafts and revision of the manuscript. V.M.M.A., R.D.G.J., and A.I.E. provided substantial contributions to the writing of the initial drafts and revisions of the manuscript for intellectual content.

\section{Data Availability Statement}

Publicly available datasets were used in this study. These can be found in http://www.google.com/trends and http://dx.doi. org/10.1016/S1474-4422(18)30403-4.

\section{Conclusions}

Google Trends data analysis has shown that there was awareness months seem to be major determinants in on-

\section{Acknowledgment}

The authors would like to thank Alvin Duke R. Sy, RN, Departtistical analysis.

References
1 Mavragani A, Ochoa G. Google trends in infodemiology and infoveillance: methodology framework. JMIR Public Health Surveill. 2019;5(2):e13439.

2 Ginsberg J, Mohebbi MH, Patel RS, Brammer L, Smolinski MS, Brilliant L. Detecting influenza epidemics using search engine query data. Nature. 2009;457(7232):1012-4.

3 Eysenbach G. Infodemiology and infoveillance: framework for an emerging set of public health informatics methods to analyze search, communication and publication behavior on the internet. J Med Internet Res. 2009;11(1):e11.

4 Patterson C. World Alzheimer report 2018 The state of the art of dementia research: new frontiers. London: Alzheimer's Disease International; 2018.

5 Watkin A, Sikdar S, Majumdar B, Richman AV. New diagnostic concepts in Alzheimer's disease. Adv Psychiatr Treat. 2013;19(4):242-9.

6 Pew Res Center. Internet/broadband fact sheet [Internet]. Washington, DC: Pew Res Center; 2021 [cited 2021 May 22]. Available from: https://www.pewresearch.org/internet/ fact-sheet/internet-broadband/. 
7 Söylemez BA, Küçükgüçlü Ö. An analysis of trends in internet searches about Dementia in Turkey. Dokuz Eylül Üniversitesi Hemşirelik Fakültesi Elektron Derg. 2020;13(1):25-9.

8 Wang HW, Chen DR, Yu HW, Chen YM. Forecasting the incidence of dementia and dementia-related outpatient visits with google trends: evidence from Taiwan. J Med Internet Res. 2015; 17(11):e264.

9 Nuti SV, Wayda B, Ranasinghe I, Wang S, Dreyer RP, Chen SI, et al. The use of Google Trends in health care research: a systematic review. PLoS One. 2014;9(10):1-49.

10 Google. Google trends [Internet]. 2021 [cited 2021 May 13]. Available from: http://www. google.com/trends.

11 GBD 2016 Dementia Collaborators. Global, regional, and national burden of Alzheimer's disease and other dementias, 1990-2016: a systematic analysis for the Global Burden of Disease Study 2016. Lancet Neurol. 20162019; 18(1):88-106

12 World Bank list of economies (June 2020) [Internet]. 2020. Available from: https://datahelpdesk.worldbank.org/knowledgebase/ articles/906519-world-bank-country-andlending-groups.

13 DeFrank TM, Haberman M, Hester J. President Ronald Reagan loses fierce battle with Alzheimer's at 93 in 2004. Daily News. 2004 Jun 4. Available from: https://www.nydailynews.com/news/national/long-struggle-gipper-dead-93-president-article-1.655972. magazine

14 Eckholm E. Robertson stirs passions with suggestion to divorce an Alzheimer's patient. The New York Times. 2011 Sep 16. Available from: https://www.nytimes.com/2011/09/17/ us/pat-robertson-remarks-on-alzheimersstir-passions.html.magazine

15 Stynes T. Biogen's Alzheimer's therapy granted FDA fast-track status. The Wall Street Journal. 2016 Sep 1. Available from: https:// www.wsj.com/articles/biogens-alzheimers- therapy-granted-fda-fast-track-status-1472769993.magazine

16 Park A. How Biogen's discontinued Alzheimer's drug got a second life. TIME. 2019 Oct 25. Available from: https://time.com/5709023/ biogen-alzheimers-drug/.magazine

17 Bates KG. B. Smith, restaurateur and lifestyle icon, dies at 70 of early onset Alzheimer's. NPR. 2020 Feb 24. Available from: https:// www.npr.org/sections/codeswitch/2020/02/2 4/808760806/b-smith-restaurateur-and-lifestyle-icon-dies-at-70-of-early-onset-alzheimers.magazine

18 Google. Google Translate [Internet]. 2021 [cited 2021 May 13]. Available from: http:// www.google.com/translate.

19 Wolters FJ, Chibnik LB, Waziry R, Anderson R, Berr C, Beiser A, et al. Twenty-seven-year time trends in dementia incidence in Europe and the United States: the Alzheimer Cohorts Consortium. Neurology. 2020 Aug 4;95(5): e519-31.

20 Ohara T, Hata J, Yoshida D, Mukai N, Nagata $\mathrm{M}$, Iwaki T, et al. Trends in dementia prevalence, incidence, and survival rate in a Japanese community. Neurology. 2017;89(20): 1925-32.

21 Li S, Yan F, Li G, Chen C, Zhang W, Liu J, et al. Is the dementia rate increasing in Beijing? Prevalence and incidence of dementia 10 years later in an urban elderly population. Acta Psychiatr Scand. 2007;115(1):73-9.

22 Alzheimer's Disease International. Dementia plans [Internet]. London: Alzheimer's Disease International; 2021 [cited 2021 Jun 11]. Available from: https://www.alzint.org/whatwe-do/policy/dementia-plans/

23 Prince M, Wimo A, Guerchet M, Ali G-C, Wu Y-T, Prina M. World Alzheimer report: the global impact of dementia. London: Alzheimer's Disease International; 2015.

24 Brigo F, Igwe SC, Ausserer H, Nardone R, Tezzon F, Bongiovanni LG, et al. Why do people google epilepsy? An infodemiologi- cal study of online behavior for epilepsy-related search terms. Epilepsy Behav. 2014;31: 67-70.

25 Brigo F, Trinka E. Google search behavior for status epilepticus. Epilepsy Behav. 2015;49: 146-9.

26 Lotto M, Aguirre PEA, Strieder AP, Cruvinel AFP, Cruvinel T. Levels of toothache-related interests of Google and YouTube users from developed and developing countries over time. PeerJ. 2019;7:e7706.

27 Brigo F, Lochner P, Tezzon F, Nardone R. Web search behavior for multiple sclerosis: an infodemiological study. Mult Scler Relat Disord. 2014;3(4):440-3.

28 Alzheimer's Disease International. World Alzheimer's month [Internet]. London: Alzheimer's Disease International; 2021 [cited 2021 May 29]. Available from: https://www. alzint.org/get-involved/world-alzheimersmonth/.

29 Kaminski M, Marlicz W, Koulaouzidis A. Googling on colonoscopy: a retrospective analysis of search engine statistics. Clin Exp Gastroenterol. 2020;13:397-405.

30 Mangono T, Smittenaar P, Caplan Y, Huang VS, Sutermaster S, Kemp H, et al. Information-seeking patterns during the COVID-19 pandemic across the United States: longitudinal analysis of Google Trends data. J Med Internet Res. 2021;23(5):1-16.

31 Fox S, Rainie L. The online health care revolution: how the web helps Americans take better care of themselves [Internet]. Washington, DC: The Pew Internet \& American Life Project; 1999. Available from: https://www.pewresearch.org/internet/2000/11/26/the-online-health-care-revolution/.

32 Johnson J. Google: search engine market share in selected countries 2021 [Internet]. Hamburg: Statista. 2021 [cited 2021 Jun 6]. Available from: https://www.statista.com/ statistics/220534/googles-share-of-searchmarket-in-selected-countries/. 